\title{
New Frontiers in Biomedical Research: A Medical Student Perspective
}

Ben Sayer. ${ }^{1}$

\section{The Experience}

When I was offered a placement in Professor Haniffa's world-leading immunology laboratory at Newcastle University, it was a one-time opportunity to gain an insight into a field that is rapidly developing. The focus of her group is mononuclear phagocytes, a family of immune cells including dendritic cells, monocytes and macrophages, in different contexts. A degree in Biomedical Sciences at St George's University of London had given me lab experience using techniques such as Western Blot, ELISA and confocal microscopy. I am now enrolled in a graduate medicine course and was keen to bring together my laboratory and clinical experience. I was particularly interested in exploring how new technologies are revolutionising biomedical research and clinical practice.

\section{New advances in biomedical research}

As part of my placement at Newcastle University's immunology lab led by Professor Haniffa, I was introduced to cutting-edge single cell technologies. I learned that high-dimensional analysis of single cells is providing new insights into biological processes and pathology. Past research has focused on morphology, physical properties and surface markers. However, this approach only facilitates study of limited cellular parameters, which introduces intrinsic bias and can lead to erroneous conclusions. Subsequent exploration of gene expression and function has been conducted on a population level, taking an average from a sample of many cells. This involved prior identification and isolation of cells using techniques such as flow cytometry, which is limited by a finite number of parameters. Study of the transcriptome (transcriptomics), which can be defined as the complete set of RNA transcripts in a cell under specific conditions, has opened up further avenues for single cell research. Single cell transcriptomics does not require a priori selection, enabling researchers to use the transcriptome of individual cells for their identification. Thus, single cell mRNA sequencing (scRNA-seq) data can be accrued to subsequently direct functional study with greater accuracy. As this approach considers many more parameters, it minimises bias within the experimental system.

\section{Bioinformatics}

A key requirement to maximise the benefits of this new research is robust bioinformatics capabilities. Bioinformatics utilises computation to convert biological data into interpretable information. Cenomics is producing increasing quantities of data which need to be stored, processed, interpreted and visualised. A suite of analysis programmes is available which are statistically-based and often incorporate machine learning algorithms. ${ }^{2}$

There is great complexity underlying the programming to manage data produced from scRNA-seq experiments. An in-depth understanding of the computing behind informatics is not necessary, but an ability to utilise and navigate algorithms to interpret data is likely to become an essential part of research in the future.

\section{Single cell RNA-sequencing in immunology}

The seminal study demonstrating the utility of scRNA-seq to define immune cell types without prior selection was performed on mouse spleen. ${ }^{3}$ More recent research in human immunology exploits scRNAseq. I will focus on two studies of human dendritic cells to demonstrate the underlying scRNA-seq experimental approach and bioinformatics concepts in interpreting scRNA-seq data. Villani et al. performed full length mRNA transcript measurement whereas See et al. measured short sequences at the $3^{\prime}$ end of mRNA transcripts which were coupled to a unique molecular identifier for each gene. ${ }^{4-5}$

Figure 1. Human blood Dendritic Cell (DC) heterogeneity delineated by single-cell RNA sequencing. t-SNE visualisation of SCRNA-seq datasets of 742 cells. Up to five top discriminators are listed next to each cluster. Each do represents an individual cell. (From Figure $1 C$ Villani et al. Single-cell RNAseq reveals new types of human blood dendritic cells, monocytes, and progenitors. Science. 2017; 356(6335). Copyright $\odot ~(2017)$ [The American Association for the Advancement of Science]. Reprinted with permission from The American Association for the Advancement of Science.

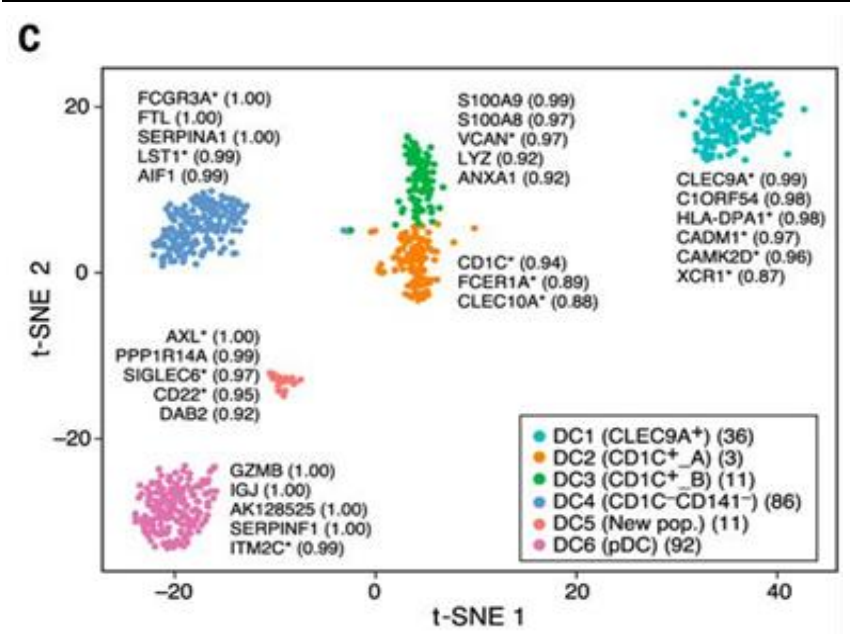

Next generation sequencing of the cDNA from single cells produces millions of short reads which need to be aligned to the reference human genome. There are several packages such as Seurat which provide an all-in-one application suite to undertake quality control, analysis, exploration and visualisation of SCRNA-seq data. One of the main analyses undertaken is to cluster cells by their respective transcriptome profiles. Cell clusters can be visually displayed using tdistributed stochastic neighbour embedding (t-SNE), which enables high-dimensional datasets to be represented in two dimensions (Figure 1). 4

Genes that best characterise each cell cluster identified can also be represented as a heat map, used by Villani et al. (Figure 2).4

\section{'St George's, University of London, London, UK}




\section{Experience}

Figure 2. Heat map display of quantified gene expression. (Figure 1D from Villani et al. Single-cell RNA-seq reveals new types of human blood dendritic cells, monocytes, and progenitors. Science. 2017; 356(6335). Copyright $(\mathrm{C}$ (2017) [The American Association for the Advancement of Science] Reprinted with permission from The American Association for the Advancement of Science).

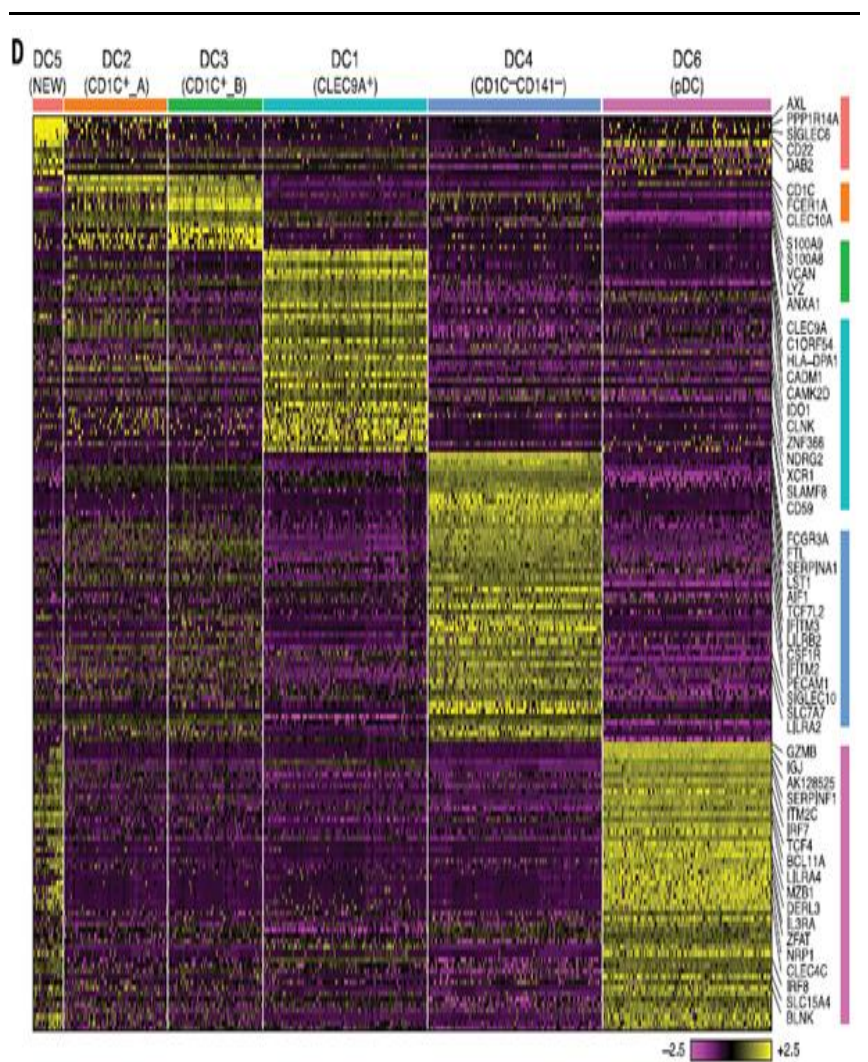

This displays the expression profile for the relevant discriminatory gene for each cell (represented by each column). High intensity expression (indicated in yellow) for the respective genes distinguish the various cell types identified from the scRNA-seq analysis.

Further understanding of cellular heterogeneity coupled with experiments exploring responses to various stimuli (e.g. viruses) and different cancer microenvironments are radically changing biomedical science. Combining this powerful methodology with proteomics and metabolomics technologies will provide unprecedented resolution to redefine disease classification, pathogenesis and identification of novel therapeutic targets. One existing example is immunotherapy for certain cancers, an area of research that is already showing remarkable promise. . $^{-8}$

\section{Conclusion}

This placement was a wonderful opportunity to engage first-hand with world-leading immunological research. I was able to learn about the changing landscape of biomedical research and the increasingly important role of bioinformatics. I would highly recommend that fellow medical students seek out similar opportunities throughout their training as biomedical research is a rapidly evolving field that will impact on how medicine will be practised in the future. 


\section{References}

1. Liang J, Cai W, Sun Z. Single-cell sequencing technologies: current and future. J Cenet Cenomics. 2014 Oct 20;41(10):513-28

2. Stegle 0 , Teichmann SA, Marioni JC. Computational and analytical challenges in single-cell transcriptomics. Nat Rev Genet. 2015 Mar;16(3):133-45.

3. Jaitin DA, Kenigsberg E, Keren-Shaul H, Elefant N, Paul F, Zaretsky I, et al. Massively parallel single cell RNA-Seq for marker-free decomposition of tissues into cell types. Science. 2014 Feb 14;343(6172):776-9.

4. Villani AC, Satija R, Reynolds G, Sarkizova S, Shekhar K, Fletcher J et al. Single-cell RNA-seq reveals new types of human blood dendritic cells, monocytes, and progenitors. Science. 2017 Apr 21;356(6335).
5. See $P$, Dutertre CA, Chen J, Cünther $P$, McGovern N, Irac SE, et al. Mapping the human $D C$ lineage through the integration of high-dimensional techniques. Science. 2017 Jun 9;356(6342).

6. van der Maaten L, Hinton G. Visualizing data using t-SNE. Journal of Machine Learning Research. 2008;9:2579-2605.

7. Kranz LM, Diken M, Haas H, Kreiter S, Loquai C, Reuter KC, et al. Systemic RNA delivery to dendritic cells exploits antiviral defence for cancer immunotherapy. Nature. 2016 Jun 16;534(7607):396-401.

8. Craciotti M, Berti C, Klok HA, Kandalaft L. The era of bioengineering: how will this affect the next generation of cancer immunotherapy? J Transl Med. 2017 Jun 19;15(1):142.

\section{Acknowledgments}

I would like to acknowledge Professor Haniffa and her team for welcoming me into their lab and for the subsequent input from Professor Haniffa to help edit and refine this article.

\section{Conflict of Interest Statement it Funding}

The Authors have no funding, financial relationships or conflicts of interest to disclose.

\section{Author Contributions}

Conceptualization: BS. Methodology: BS. Writing - Original Draft: BS. Writing - Review at Editing: BS. Visualization: BS.

Cite as:

Sayer B. New Frontiers in Biomedical Research: A Medical Student Perspective. Int J Med Students. 2018 Sep-Dec;6(3):120-122. 